\title{
Generalized composed radial epiderivatives
}

\author{
Gonca Inceoglu \\ Department of Mathematics and Science Education, Anadolu University, Turkey
}

Received: 24 November 2017, Accepted: 29 January 2018

Published online: 7 March 2018.

\begin{abstract}
In this paper, the generalized composed radial epiderivative for set-valued maps is introduced and some of its properties are investigated. Existence conditions for the generalized composed radial epiderivative are established.
\end{abstract}

Keywords: Radial cone, generalized composed radial epiderivative, set-valued optimization.

\section{Introduction}

In the last thirty years the notion of derivatives or epiderivatives has been formulated in different ways. (see [1,2,7,8, 10, $13,19,23,24,25,28,31])$. Aubin first introduced the notion of the contingent derivative for set-valued map by using the contingent cone [1]. Corley established the sufficient and necessary optimality conditions for set-valued optimization problems by virtue of the concept of contingent and circatangent derivative [13]. The contingent derivative play an important role for set-valued optimization problems. But, necesary and sufficient optimality conditions do not coincide unified under standart assumptions. To overcome the difficulty, another of differentiability concept which is based on using epigraphs of set-valued maps was proposed Jahn and Rauh [23].

Kasımbeyli introduced in [24] the notion of the radial epiderivative of a nonconvex set-valued map. This definition of the radial epiderivative given by Kasımbeyli is different from that of Flores- Bazan [7] and is similar to the definition of the contingent epiderivative given by Jahn and Rauh [23]. He derived the formulation of optimality conditions in the single valued and set-valued optimization without convexity assumption and investigated relationships between this kind of epiderivative and weak subdifferentials and directional derivatives for real-valued nonconvex functions.

Kasımbeyli and Inceoglu introduced in [25] the notion of generalized radial epiderivative for set-valued maps and investigated existence conditions for generalized radial epiderivative. They established the relationship between the radial epiderivative and the generalized radial epiderivative. By using the generalized radial epiderivative, Kasimbeyli and Inceoglu presented the necessary and sufficient optimality conditions for set-valued optimization.

Recently, there has been an increasing interest in second-order and higher-order optimality research for set-valued map $[3,4,5,6,9,11,12,14,15,16,21,22,27,20,26,29,30,32]$. Jahn et al. proposed the second-order epiderivatives in terms of the second-order contingent set [21], introduced by Aubin and Frankowska [2]. They obtained the second-order optimality conditions by using these dervatives in set-valued optimization.It can be seen that a second-order contingent set, introduced by Aubin and Frankowska [2], and a second-order asymptotic contingent cone, introduced by Penot [30], play a important role in establishing second-order optimality conditions. Li et al. proposed a generalized second-order composed contingent epiderivative for a set-valued map and investigated some of its properties. By virtue of the generalized second-order composed contingent epiderivative, they also establised a unified second-order sufficient and 
necessary optimality conditions for set-valued optimization [29]. Isac and Khan employed the second-orer optimality conditions in set-valued optimization problems, using which they introduced new kind of second-order tangent epiderivative [16].

Anh and Khanh introduced the higher-order radial sets and corresponding derivatives. They proposed their properties and basic calculus rules. They established both necessary and sufficient higher-order conditions for weak efficiency in set-valued vector optimization problem [4]. Anh and Khanh gived both necessary and sufficient higher-order conditions for various kinds of proper solutions to nonsmooth vector optimization problem in terms of higher-order radial sets and radial derivatives [5]. In [25], Inceoglu introduce the concepts of second-order radial epiderivative and second-order generalized radial epiderivative for nonconvex set-valued maps. They also investigate in [25] some of their properties and give existence theorems for the second-order generalized radial epiderivatives. In [25], Inceoglu propose second-order optimality conditions by using second-order radial epiderivatives.It is wort noting that higher-order radial derivative or radial epiderivative by a higher-order radial set, in general, is not a cone and a convex set. Therefore, there are some difficulties in studying higher-order optimality conditions for set-valued optimization problems.

Motivated by this problem, we intend to give a new generalized second-order composed radial epiderivatives for set-valued maps and investigate some of its properties. By using this concept, we give a unified second-order sufficient and necessary optimality conditions for set-valued optimization problems, which is a generalization of the corresponding in [25]. This paper is divided into two sections. In Section 2 , we give the second-order radial epiderivatives and prove the existence conditions of one of them.

\section{Preliminaries}

Throughout this paper, Let $\left(X,\|\cdot\|_{X}\right)$ and $\left(Y,\|\cdot\|_{Y}\right)$ real normed spaces and $S$ be a nonempty subset of $X$. Let $C \subset Y$ a pointed, closed and convex cone with apex at the origin and a nonempty interior int $C$, and let $Y$ be partially ordered by $C$. Let $F: S \rightrightarrows Y$ be a set-valued map. Let a pair $(\bar{x}, \bar{y}) \in \operatorname{graph}(F)$ be given. In this section, we recall the concept of the radial epiderivative and the generalized radial epiderivative introduced by Kasımbeyli [24], and Kasımbeyli and Inceoğlu [25], respectively, together with some standart notions.

Definition 1. Let $U$ be a nonempty subset of a real normed space $\left(Z,\|\cdot\|_{Z}\right)$, and let $\bar{z} \in \operatorname{cl}(U)$ (closure of $U$ ) be a given element. The closed radial cone $R(U, \bar{z})$ of $U$ at $\bar{z} \in c l(U)$ is the set of all $z \in Z$ such that there are $\lambda_{n}>0$ and a sequence $\left(z_{n}\right)_{n \in \mathbb{N}} \subset Z$ with $\lim _{n \rightarrow \infty} z_{n}=z$ so that $\bar{z}+\lambda_{n} z_{n} \in U$, for all $n \in \mathbb{N}$.

Note that the closed radial cone can equivalently be also defined as the following definition.

Definition 2. Let $U$ be a nonempty subset of a real normed space $\left(Z,\|\cdot\|_{Z}\right)$, and let $\bar{z} \in \operatorname{cl}(U)$ (closure of $U$ ) be a given element. The closed radial cone $R(U, \bar{z})$ of $U$ at $\bar{z} \in c l(U)$ is the set of all $z \in Z$ such that there are $\lambda_{n}>0$ and a sequence $\left(z_{n}\right)_{n \in \mathbb{N}} \subset U$ with $\lim _{n \rightarrow \infty} \lambda_{n}\left(z_{n}-\bar{z}\right)=z$.

It follows from these definitions that

$$
R(U, \bar{z})=\operatorname{cl}(\text { cone }(U-\bar{z}))
$$

where cone denotes the conic hull of a set, which is the smallest cone containing $U-\bar{z}$.

Definition 3. Let $F: X \rightrightarrows Y$ be a set-valued map.

(i) The set

$$
\operatorname{graph}(F)=\{(x, y) \in X \times Y \mid y \in F(x)\}
$$

is called the graph of $F$; 
(ii) The set

$$
\operatorname{dom}(F)=\{x \in X \mid F(x) \neq \emptyset\}
$$

is called the domain of $F . F$ is said to be proper if $\operatorname{dom}(F) \neq \emptyset$;

(iii) Let $Y$ be partially ordered by a proper, convex, and pointed cone $C \subset Y$. The set

$$
\operatorname{epi}(F)=\{(x, y) \in X \times Y \mid y \in F(x)+C\}
$$

is called the epigraph of $F$.

Now we give the definition of the radial epiderivative given by Kasimbeyli without convexity and boundedness [24].

Definition 4. Let $F: S \rightrightarrows Y$ be a set-valued map. Let a pair $(\bar{x}, \bar{y}) \in \operatorname{graph}(F)$ be given. A single-valued map $D_{r} F(\bar{x}, \bar{y})$ : $X \rightarrow Y$ whose epigraph equals the radial cone to the epigraph of $F$ at $(\bar{x}, \bar{y})$ i.e.

$$
\operatorname{epi}\left(D_{r} F(\bar{x}, \bar{y})\right)=R(\operatorname{epi}(F),(\bar{x}, \bar{y}))
$$

is called radial epiderivative of $F$ at $(\bar{x}, \bar{y})$.

Definition 5. Let $F: X \rightrightarrows Y$ be a set valued map and let $(\bar{x}, \bar{y}) \in \operatorname{graph}(F)$. A set valued map $D_{R} F(\bar{x}, \bar{y}): X \rightrightarrows Y$ whose graph coincides with the contingent cone to graph of $F$ at $(\bar{x}, \bar{y})$, that is

$$
\operatorname{graph}\left(D_{r} F(\bar{x}, \bar{y})\right)=R(\operatorname{graph}(F),(\bar{x}, \bar{y}))
$$

is called radial derivative of $F$ at $(\bar{x}, \bar{y})[7,31]$.

To give the definition of the generalized radial epiderivative, we recall the minimality concept.

Definition 6. Let $\left(Y,\|\cdot\|_{Y}\right)$ be a real normed space partially ordered by a convex cone $C \subset Y$. Let D be a subset of $Y$ and let $\bar{y} \in D$.

(i) The element $\bar{y}$ is said to be a minimal element of $D$, if $D \cap(\{\bar{y}\}-C)=\{\bar{y}\}$.

(ii) Let the ordering cone have a nonempty interior int $(C)$. The element $\bar{y}$ is said to be a weakly minimal element of $D$, if $D \cap(\{\bar{y}\}-\operatorname{int}(C))=\emptyset$. The set of all minimal, weakly minimal elements of $D$ with respect to the ordering cone $C$ is denoted by $\operatorname{Min}(D, C), w-\min (D, C)$, respectively.

Definition 7. A set valued map $D_{g r} F(\bar{x}, \bar{y}): S-\{\bar{x}\} \rightrightarrows Y$ is called the generalized radial epiderivative of $F$ at $(\bar{x}, \bar{y}) ;$ if

$$
D_{g r} F(\bar{x}, \bar{y})(x)=\operatorname{Min}\{y \in Y \mid(x, y) \in R(\operatorname{epi}(F),(\bar{x}, \bar{y}))\}, \forall x \in X
$$

where MinD is the set of all minimal elements of D [25, Definition 8].

Notice that for some $x \in S-\{\bar{x}\}$ the set $\{y \in Y \mid(x, y) \in R($ epi $(F),(\bar{x}, \bar{y}))\}$ may be empty. In this case we have $D_{g r} F(\bar{x}, \bar{y})(x)=\emptyset$

\section{Generalized composed radial epiderivatives}

In this section we introduce a generalized composed radial epiderivative for set-valued maps, and give some of properties of its and existence theorems. The following concept extends a characterization of Definition 7 given by Kasımbeyli and Inceoğlu [25]. 
Definition 8. Let $F: S \rightrightarrows Y$ be a set-valued map and $(\bar{x}, \bar{y}) \in \operatorname{graph} F$, and $(\bar{u}, \bar{v}) \in X \times Y$. The generalized second-order composed radial epiderivative of $F$ at $(\bar{x}, \bar{y})$ in the direction $(\bar{u}, \bar{v})$ is the set-valued map $D_{\text {grc }}^{2} F(\bar{x}, \bar{y}, \bar{u}, \bar{v}): X \rightrightarrows Y$ defined by

$$
D_{g r c}^{2} F(\bar{x}, \bar{y}, \bar{u}, \bar{v})=\operatorname{Min}\{y \in Y \mid(x, y) \in R(R(\operatorname{epi} F,(\bar{x}, \bar{y})),(\bar{u}, \bar{v}))\} .
$$

Proposition 1. $R(R(S, \bar{z}), w)=$ clcone $($ cone $(S-\bar{z})-w)$.

Proof. Let $K=$ cone $(S-\bar{z})$, then

$$
\begin{gathered}
R(K, w)=\text { clcone }(K-w) \\
R(K, w)=R(c l K, w)=R(R(S, \bar{z}), w) .
\end{gathered}
$$

Lemma 1. Let $F: S \rightrightarrows Y$ be a set-valued map and $(\bar{x}, \bar{y}) \in \operatorname{graph} F$, and $(\bar{u}, \bar{v}) \in X \times Y$. Then we have the following statement:

$$
R(R(\operatorname{epiF},(\bar{x}, \bar{y})),(\bar{u}, \bar{v}))+\left\{0_{X}\right\} \times C=R(R(\operatorname{epiF},(\bar{x}, \bar{y})),(\bar{u}, \bar{v}))
$$

Proof. We need to prove that

$$
R(R(e p i F,(\bar{x}, \bar{y})),(\bar{u}, \bar{v}))+\left\{0_{X}\right\} \times C \subset R(R(e p i F,(\bar{x}, \bar{y})),(\bar{u}, \bar{v}))
$$

For every $(x, y) \in R(R($ epiF, $(\bar{x}, \bar{y})),(\bar{u}, \bar{v}))+\left\{0_{X}\right\} \times C$, there exist $(\hat{x}, \hat{y}) \in R(R($ epiF, $(\bar{x}, \bar{y})),(\bar{u}, \bar{v}))$ and $c \in C$ such that

$$
(x, y)=(\hat{x}, \hat{y})+\left(0_{X}, c\right)=(\hat{x}, \hat{y}+c)
$$

Since $(\hat{x}, \hat{y}) \in R(R($ epiF, $(\bar{x}, \bar{y})),(\bar{u}, \bar{v}))$, by the definiton of radial cone, by the definition of radial cone, there are sequence $\left(x_{n}, y_{n}\right) \rightarrow(\hat{x}, \hat{y})$ and $t_{n}>0$ such that $(\bar{u}, \bar{v})+t_{n}\left(x_{n}, y_{n}\right) \in R($ epiF, $(\bar{x}, \bar{y})), \forall n \in \mathbb{N}$. Moreover, $\forall n \in \mathbb{N}$, there exist sequences $\left(x_{n}^{k}, y_{n}^{k}\right) \rightarrow(\bar{u}, \bar{v})+t_{n}\left(x_{n}, y_{n}\right)$ and $t_{n}^{k}>o$ such that $(\bar{x}, \bar{y})+t_{n}^{k}\left(x_{n}^{k}, y_{n}^{k}\right) \in e p i F, \forall k \in \mathbb{N}$. Then we have

$$
\bar{y}+t_{n}^{k} y_{n}^{k} \in F\left(\bar{x}+t_{n}^{k} x_{n}^{k}\right)+C, \quad \forall n, k \in \mathbb{N}
$$

Since $C$ is a cone and $c \in C$, together with (3), we have

$$
\bar{y}+t_{n}^{k}\left(y_{n}^{k}+t_{n} c\right)=\bar{y}+t_{n}^{k} y_{n}^{k}+t_{n}^{k} t_{n} c \in F\left(\bar{x}+t_{n}^{k} x_{n}^{k}\right)+C, \quad \forall n, k \in \mathbb{N},
$$

that is, $(\bar{x}, \bar{y})+t_{n}^{k}\left(x_{n}^{k}, y_{n}^{k}+t_{n} c\right) \in$ epiF $\forall n, k \in \mathbb{N}$. Since $\left(x_{n}^{k}, y_{n}^{k}\right) \rightarrow(\bar{u}, \bar{v})+t_{n}\left(x_{n}, y_{n}\right)$ as $k \rightarrow \infty$. Thus $(\bar{u}, \bar{v})+t_{n}\left(x_{n}, y_{n}+c\right) \in R($ epiF,$(\bar{x}, \bar{y})), \forall n \in \mathbb{N}$. Simultaneously, $\left(x_{n}, y_{n}+c\right) \rightarrow(\hat{x}, \hat{y}+c)$ since $\left(x_{n}, y_{n}\right) \rightarrow(\hat{x}, \hat{y})$ as $n \rightarrow \infty$, that is, $(x, y)=(\hat{x}, \hat{y}+c) \in R(R(e p i F,(\bar{x}, \bar{y})),(\bar{u}, \bar{v}))$. This completes the proof.

Now, we define a strictly positive homogeneous and subadditive map and then show under appropriate assumption that the generalized second-order composed radial epiderivative is strictly positive homogeneous and subadditive [10,25].

Definition 9. A map $F: S \rightrightarrows Y$ is called

(a) strictly positive homogeneous if $F(\alpha x)=\alpha F(x)$ for all $\alpha>0$ and all $x \in X$.

(b) subadditive

$$
F\left(x_{1}\right)+F\left(x_{2}\right) \subset F\left(x_{1}+x_{2}\right)+C .
$$

If the properties a) holds with $\alpha \geq 0$ and b) holds, $F$ is called sublinear [10].

Theorem 1. Let for every $x \in X$, the generalized composed radial epiderivative $D_{g r c}^{2} F(\bar{x}, \bar{y}, \bar{u}, \bar{v})(x) \neq \emptyset$. Then $D_{g r c}^{2} F(\bar{x}, \bar{y}, \bar{u}, \bar{v})$ is strictly positive homogeneous. Moreover, if $R(R(e p i F,(\bar{x}, \bar{y})),(\bar{u}, \bar{v}))$ is a convex cone and the set

$$
\hat{G}(x)=\{y \in Y \mid(x, y) \in R(R(e p i F,(\bar{x}, \bar{y})),(\bar{u}, \bar{v}))\}
$$


fulfills the domination property for all $x \in X$, then $D_{g r c}^{2} F(\bar{x}, \bar{y}, \bar{u}, \bar{v})$ is subadditive.

Proof. Firstly, we prove the strictly positive homogenity. For every $\lambda>0$ ve $x \in X$, we have

$$
\begin{aligned}
D_{g r c}^{2} F(x, y, u, v)(\lambda x) & =\operatorname{Min}\{y \in Y \mid(\lambda x, y) \in R(R(\text { epiF },(\bar{x}, \bar{y})),(\bar{u}, \bar{v}))\} \\
& =\operatorname{Min}\{\lambda u \in Y \mid(\lambda x, \lambda u) \in R(R(\text { epiF },(\bar{x}, \bar{y})),(\bar{u}, \bar{v}))\} \\
& =\lambda \operatorname{Min}\{u \in Y \mid(x, u) \in R(R(\text { epiF },(\bar{x}, \bar{y})),(\bar{u}, \bar{v}))\} \\
& =\lambda D_{g r c}^{2} F(x, y, u, v)(x) .
\end{aligned}
$$

For every $x_{1}, x_{2} \in X$ and $y_{1} \in D_{g r c}^{2} F(\bar{x}, \bar{y}, \bar{u}, \bar{v})\left(x_{1}\right), y_{2} \in D_{g r c}^{2} F(\bar{x}, \bar{y}, \bar{u}, \bar{v})\left(x_{2}\right)$, let $\left(x_{1}, y_{1}\right) \in R(R($ epi $F,(\bar{x}, \bar{y})),(\bar{u}, \bar{v}))$ and $\left(x_{2}, y_{2}\right) \in R(R(e p i F,(\bar{x}, \bar{y})),(\bar{u}, \bar{v}))$. Since $R(R(e p i F,(\bar{x}, \bar{y})),(\bar{u}, \bar{v}))$ is a closed and convex cone, we have

$$
\frac{1}{2}\left(x_{1}, y_{1}\right)+\frac{1}{2}\left(x_{2}, y_{2}\right) \in R(R(e p i F,(\bar{x}, \bar{y})),(\bar{u}, \bar{v}))
$$

that is,

$$
\left(x_{1}+x_{2}, y_{1}+y_{2}\right) \in R(R(e p i F,(\bar{x}, \bar{y})),(\bar{u}, \bar{v})) .
$$

Thus, we have

$$
D_{g r c}^{2} F(\bar{x}, \bar{y}, \bar{u}, \bar{v})\left(x_{1}\right)+D_{g r c}^{2} F(\bar{x}, \bar{y}, \bar{u}, \bar{v})\left(x_{2}\right) \subset \hat{G}\left(x_{1}+x_{2}\right) .
$$

It follows from the domination property and definition of the generalized second-order composed radial epiderivative that

$$
\hat{G}\left(x_{1}+x_{2}\right) \subset \operatorname{Min} \hat{G}\left(x_{1}+x_{2}\right)+C=D_{g r c}^{2} F(\bar{x}, \bar{y}, \bar{u}, \bar{v})\left(x_{1}+x_{2}\right)+C
$$

Therefore, we have

$$
D_{g r c}^{2} F(\bar{x}, \bar{y}, \bar{u}, \bar{v})\left(x_{1}\right)+D_{g r c}^{2} F(\bar{x}, \bar{y}, \bar{u}, \bar{v})\left(x_{2}\right) \subset D_{g r c}^{2} F(\bar{x}, \bar{y}, \bar{u}, \bar{v})\left(x_{1}+x_{2}\right)+C
$$

Definition 10. Let $Y$ be a partially ordered by a pointed, closed, and convex cone $C \subset Y$ wiht apex at origin and a nonempty interior int $(C)$.

(a) The cone $C$ is Daniell, if any decreasing sequence in $Y$ having a lower bound, converges to its infimum.

(b) A subset of $D$ of $Y$ is said to be minorized, if there is a $y \in Y$ so that $D \subset\{y\}+C$.

(c) The domination property is said to be hold for a sunset $D$ of $Y$ if $D \subset \min (D, C)+C$ [28].

Now we need to establish an existence theorem for the generalized second-order radial epiderivative.

Theorem 2. Let $F: S \rightrightarrows Y$ be a set-valued map, $\bar{x} \in S, \bar{y} \in F(\bar{x})$, and $(\bar{u}, \bar{v}) \in X \times Y$. Additionally, let $C$ be Daniell and let $C$ be a closed pointed convex cone. Let for every $x \in S$

$$
\hat{G}(x)=\{y \in Y \mid(x, y) \in R(R(e p i F,(\bar{x}, \bar{y})),(\bar{u}, \bar{v}))\}
$$

be minorized. Then for all $x \in S$ the generalized second-order composed radial epiderivative $D_{g r c}^{2} F(\bar{x}, \bar{y}, \bar{u}, \bar{v})$ is exists.

Proof. Since the radial cone is always closed in a normed space, then for every $x \in S$ the set $\hat{G}(x)$ is and closed and minorized. From the existence theorems of minimal elements (see proposition 3.16. page in Luc) min $\hat{G}(x)$ is nonempty, i.e. $D_{g r c}^{2} F(\bar{x}, \bar{y}, \bar{u}, \bar{v})$ is well defined.

Theorem 3. Let $F: S \rightrightarrows Y$ be a set-valued map, $(\bar{x}, \bar{y}) \in \operatorname{graph}(F)$, and $(\bar{u}, \bar{v}) \in X \times Y$. If the generalized second-order composed radial epiderivative $D_{g r c}^{2} F(\bar{x}, \bar{y}, \bar{u}, \bar{v})$ exists and the set

$$
\hat{G}(x)=\{y \in Y \mid(x, y) \in R(R(e p i F,(\bar{x}, \bar{y})),(\bar{u}, \bar{v}))\}
$$


fulfills the domination property for all $x \in X$, then

$$
\operatorname{epi}\left(D_{g r c}^{2} F(\bar{x}, \bar{y}, \bar{u}, \bar{v})\right)=R(R(\operatorname{epi} F,(\bar{x}, \bar{y})),(\bar{u}, \bar{v}))
$$

Proof. For every $(x, y) \in \operatorname{epi}\left(D_{g r c}^{2} F(\bar{x}, \bar{y}, \bar{u}, \bar{v})\right)$ there exist $\bar{y} \in D_{g r c}^{2} F(\bar{x}, \bar{y}, \bar{u}, \bar{v})(x)$ and $c \in C$, such that $y=\bar{y}+c$. Since $\bar{y} \in D_{g r c}^{2} F(\bar{x}, \bar{y}, \bar{u}, \bar{v})(x)$, it follows from definition of the $D_{g r c}^{2} F$ that $(x, \bar{y}) \in R(R(e p i F,(\bar{x}, \bar{y})),(\bar{u}, \bar{v}))$. Then we get

$$
(x, y)=(x, \bar{y})+\left(0_{X}, c\right) \in R(R(e p i F,(\bar{x}, \bar{y})),(\bar{u}, \bar{v}))+\left\{0_{X}\right\} \times C=R(R(e p i F,(\bar{x}, \bar{y})),(\bar{u}, \bar{v}))
$$

which implies

$$
\operatorname{epi}\left(D_{g r c}^{2} F(\bar{x}, \bar{y}, \bar{u}, \bar{v})\right) \subset R(R(\operatorname{epi} F,(\bar{x}, \bar{y})),(\bar{u}, \bar{v})) \text {. }
$$

Conversely, suppose that $(x, y) \in R(R($ epiF,$(\bar{x}, \bar{y})),(\bar{u}, \bar{v}))$, then $y \in \hat{G}(x)$. By the domination property, there exists some $\bar{y} \in \operatorname{Min} \hat{G}(x)$, such that $y \in\{\bar{y}\}+C$. By the definition of $D_{g r c}^{2} F$, we have

$$
\operatorname{Min} \hat{G}(x)=D_{g r c}^{2} F(\bar{x}, \bar{y}, \bar{u}, \bar{v})(x) .
$$

Thus $y \in D_{g r c}^{2} F(\bar{x}, \bar{y}, \bar{u}, \bar{v})(x)+C$, that is, $(x, y) \in \operatorname{epi}\left(D_{g r c}^{2} F(\bar{x}, \bar{y}, \bar{u}, \bar{v})\right)$. Then we can conclude that

$$
\operatorname{epi}\left(D_{g r c}^{2} F(\bar{x}, \bar{y}, \bar{u}, \bar{v})\right)=R(R(\operatorname{epi} F,(\bar{x}, \bar{y})),(\bar{u}, \bar{v}))
$$

\section{Conclusion}

In this article, we introduce a new second-order radial epiderivative by taking radial epiderivative of a radial epiderivative. We obtain the some properties of these second-order radial epideivative.

\section{Competing interests}

The authors declare that they have no competing interests.

\section{Authors' contributions}

All authors have contributed to all parts of the article. All authors read and approved the final manuscript.

\section{References}

[1] Aubin, J.P.,1981, Contingent Derivatives of Set-Valued Maps and Existence of Solutions to Nonlinear Inclusions and Differential Inclusions. In: Nachbin, L (ed.) Mathematics Analysis and Applications, part A, 160-229, Academic Press, New York.

[2] Aubin, J.P., Frankowska, H., 1990, Set Valued Analysis,Birkhauser, Boston.

[3] Aghezzaf, B. and Hachimi, M., 1999, Second Order Optimality Conditions in Multiobjective Optimization Problems,J. Optim. Theory Apply., 102,1,37-50.

[4] Anh, N.L.H., and Khanh, P.Q., 2013, Higher-Order Optimality Conditions in Set-Valued optimization Using Radial Sets and Radial Derivatives. J. Glob Optim.,56,2,519-536.

[5] Anh, N.L.H. and Khanh, P.Q., 2014, Higher-Order optimality Conditions for Proper Efficiency in Nonsmooth Vector Optimization Using Radial Sets and Radial Derivatives,J. Glob Optim., 58,4, 693-709.

[6] Anh, N.L.H. Khanh, P.Q. and Tung, L.T., 2011, Higher-Order Radial Derivatives and Optimality Conditions in Nonsmooth Vector Optimization, Nonlinear Anal.Theory Meth.Appl.,74,7365-7379. 
[7] Bazan, F.F., 2001, Optimality Conditions in Nonconvex Set-Valued Optimization, Mathematical Methods of Operations Research,53, 403-417.

[8] Bazan, F.F., 2003, Radial Epiderivatives and Asymptotic Functions in Nonconvex Vector Optimization, SIAM J. Optimization, 14,284-305.

[9] Bigi, G. and Castellani, M.,2000, Second Order Optimality Conditions for Differentiable Multiobjective Problems, RARIO Operations Research, 34,411-426.

[10] Chen, G.Y. and Jahn, J., 1998, Optimality Conditions for Set-Valued Optimization Problems, Mathematical Methods of Operations Research, 48,187-200.

[11] Cambini, A. and Martein, L., 2002, First and Second Order Optimality Conditions in Vector Optimization,Journal of Statistics and Management Systems,5,295-319.

[12] Cambini, A., Martein, L. and Vlach,M., 1999, Second Order Tangent Sets and Optimality Conditions, Matematica Japonica, 49,451-461.

[13] Corley, H.W., 2001, Optimality Conditions for Maximization in Partially Ordered Linear Spaces, J. Optim. Theory Apply., 53, 403-417.

[14] Giorgi, G., Jimenez, B. and Novo, V., 2010, An Overview of Second Order Tangent Sets and Their Application to Vector Optimization, SeMA Journal,52, 1, 73-96.

[15] Gutierrez,C., Jimenez,B. and Novo, V., 2009, New Second-Order Directional Derivative and Optimality Conditions in Scalar and Vector Optimization, J. Optim. Theory Appl., 142,85-106.

[16] Isac, G., and Khan, A.A., 2009, Second-Order Optimality Conditions In set-Valued Optimizaiton By A New Tangential Derivative, Acta Matematica Vietnamica, 34, 81-90.

[17] Ha,T.D.X., 2009, Optimality conditions for several types of efficient solutions of set-valued optimization problems,in: P. Pardolos, Th.M. Rassis, A.A. Khan (Eds.), Nnlinear Analysis and Variational Problems, Springer, p.305-324(Chapter 21).

[18] Hachimi, M. and Aghezzaf, B., 2007, New Results on Second-Order Optimality Conditions in Vector Optimization Problems, J. Optim. Theory Appl.,135,117-133.

[19] Jahn,J., 1986, Mathematical vector optimization in partially ordered linear space, Peter Lang, Frankfurt.

[20] Jimenez, B. and Novo, V., 2003, Second Order Necessary Conditions in Set Constrained Differentiable Vector Optimization, Mathematical Methods of Operations Research, 58,299-317.

[21] Jahn,J., Khan,A.A., and Zeillinger, P., 2005, Second Order Optimality Conditions in Set Optimization, J. Optim. Theory Apply., 125,2,331-347.

[22] Jimenez, B. and Novo, V.,2004, Optimality Conditions in Differentiable Vector Optimization via Second-Order Tangent Sets, Appl. Math. Optim., 49,123-144

[23] Jahn, J. and Rauh, R.,1997, Contingent Epiderivatives and Set-Valued Optimization Mathematical Methods of Operations Research, 46,193-211.

[24] Kasımbeyli,R.,2009 Radial Epiderivatives and Set-Valued Optimization, Optimization,58,5,519-532.

[25] Kasımbeyli,R. and İnceoğlu, G.,2010, Optimality Conditions viaGeneralized Radial Epiderivatives in Nonconvex Set-Valued Optimization, In: R. Kasımbeyli, C. Dinçer, S. Özpeynirci and L. Sakalauskas (Eds.) Selected papers. 24th Mini EURO Conference on Continuous Optimization and Information-Based Technologies in the Financial Sector (24th MEC EurOPT 2010), June 23-26, 2010, Izmir University of Economics, Izmir, Turkey, ISBN: 978-9955-28-597-7, Vilnius ’Technika”, p. 148-154.

[26] Kalashnikov, V., Jadamba, B. and Khan,A.A., 2006, First and Second- Order Optimality Condition in Set-Optimization, In Optimization with Multivalued Mappings, Edited by: Dempe, S and Kalashnikov, V. , Berlin, Heidelberg: Springer Verlag, 265276.

[27] Khan, A.A. and Tammer, C., 2013, Second Order Optimality Conditions in Set-Valued Optimization via Asymptotic Derivatives, Optimization, 62,6,743-758.

[28] Luc, D.T., 1991, Theory of Vector Optimization, Springer, Berlin.

[29] Li, S.J., Zhu, S.K. and Teo, K.L., 2012, New Generalized Second-Order Contingent Epideriv. and Set- Valued Optmization Problem, J. Optim. Theory Apply.,152, 3, 587-60.

[30] Penot, J.P., 1999, Second-Order Conditions for Optimization Problems Constraints, SIAM Journal on Optimization, 37, $303-318$.

[31] Taa, A., 1998, Set-valued derivatives of multifunctions and optimality conditions, Num. Func. anal. optim. 19, 121-140.

[32] Ward, D., 1993, Calculus for Parabolic Second-Order Derivatives. Set Valued Analysis, 1,213-246. 